\title{
The "Lid" in the Streptococcus Pneumoniae SrtC1 Sortase Adopts a Rigid Structure that Regulates Substrate Access to the Active Site
}

\author{
Alex W. Jacobitz ${ }^{\dagger, * \star}$, Emmanuel B. Naziga ${ }^{\ddagger,},{ }^{\star \star}$, Sung Wook $\mathrm{Yi}^{\dagger}$, Scott A. McConnell ${ }^{\dagger}$, Robert \\ Peterson ${ }^{\dagger}$, Michael E. Jung ${ }^{\dagger}$, Robert T. Clubb ${ }^{\dagger},{ }^{*}$, and Jeff Wereszczynski ${ }^{\ddagger},{ }^{*}$ \\ †Department of Chemistry and Biochemistry and the UCLA-DOE Institute of Genomics and \\ Proteomics, University of California, Los Angeles, 611 Charles E. Young Drive East, Los Angeles, \\ CA $90095-1570$ \\ ¥Department of Physics and Center for Molecular Study of Condensed Soft Matter, Illinois \\ Institute of Technology, 3440 S Dearborn St, Chicago, IL 60616
}

\begin{abstract}
Many species of Gram-positive bacteria use sortase enzymes to assemble long, proteinaceous pili structures that project from the cell surface to mediate microbial adhesion. Sortases construct highly stable structures by catalyzing a transpeptidation reaction that covalently links pilin subunits together via isopeptide bonds. Most Gram-positive pili are assembled by class $\mathrm{C}$ sortases that contain a "lid", a structurally unique $\mathrm{N}$-terminal extension that occludes the active site. It has been hypothesized that the "lid" in many sortases is mobile, and thus capable of readily being displaced from the enzyme to facilitate substrate binding. Here, we show using NMR dynamics measurements, in vitro assays, and molecular dynamics simulations that the lid in the class $\mathrm{C}$ sortase from Streptococcus pneumoniae ( $\mathrm{SrtC1}$ ) adopts a rigid conformation in solution that is devoid of large magnitude conformational excursions that occur on mechanistically relevant timescales. Additionally, we show that point mutations in the lid induce dynamic behavior that correlates with increased hydrolytic activity and sorting signal substrate access to the active site cysteine residue. These results suggest that the lid of the $S$. pneumoniae $\mathrm{SrtC1}$ enzyme has a negative regulatory function, and imply that a significant energetic barrier must be surmounted by as of yet unidentified factors to dislodge it from the active site to initiate pilus biogenesis.
\end{abstract}

\section{Graphical Abstract}

\footnotetext{
*Corresponding Authors. rclubb@mbi.ucla.edu, Tel: +1 (310) 206 2334; jwereszc@iit.edu, Tel: +1 (312) 5673322.

Alex Jacobitz and Emmanuel B. Naziga contributed equally to this work.

Author Contributions

All authors contributed to the work in this manuscript.

The authors declare no competing financial interest.

ASSOCIATED CONTENT

Supporting Information

Additional experimental and computational details, including several figures referred to in the main article, as well as a movie describing the "lid" opening process are provided in the associated Supplementary Information (SI).
} 


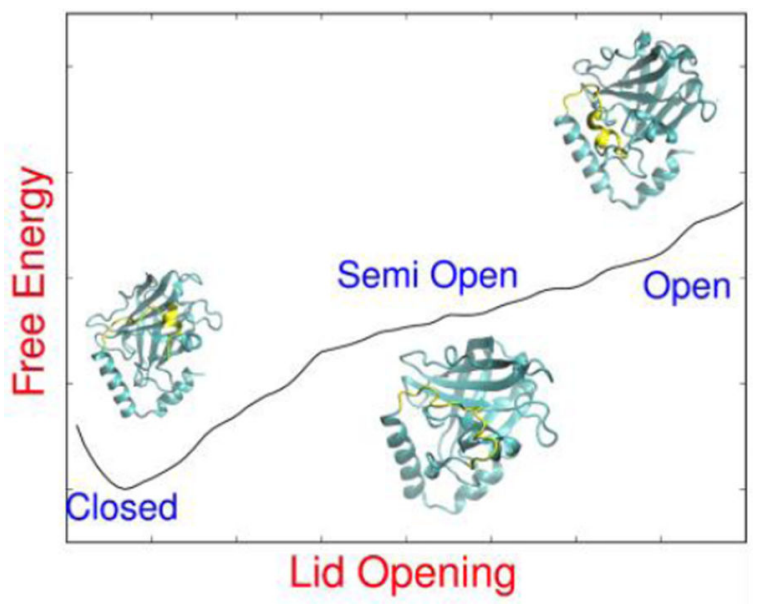

\section{Introduction}

Gram-positive bacteria use class $\mathrm{C}$ sortases to construct pili, long filamentous protein fibers that project from the cell surface ${ }^{1-3}$. These pili provide a preliminary point of attachment to host cells, acting as a grappling hook that allows the bacterium to swing closer to its target thereby facilitating the formation of close-range adhesive interactions via auxiliary surface anchored adhesive proteins ${ }^{3}$. Pili also participate in immune system evasion ${ }^{4}$, biofilm formation $^{5,6}$ and have great potential as vaccine components due to their ability to elicit an immune response $\mathrm{e}^{7-9}$. These diverse roles have led to the recognition that pili function as key virulence factors in pathogenic microbes, making their assembly process an exciting new antibacterial target ${ }^{3,10-13}$.

Pilus assembling class-C sortases are associated with the extracellular membrane and catalyze a transpeptidation reaction that covalently joins pilin subunits together via isopeptide bonds. In this reaction, the enzyme recognizes a five residue sorting signal motif that is located near the C-terminus of the pilin protein and is followed by a transmembrane helix and a positively charged cytoplasmic anchoring domain. This sorting signal is generally of the form LP XTG, where the Pro and Thr residues are the most highly conserved, $X$ can be any amino acid, and the first and last residue often confer specificity to the individual sortase ${ }^{14-16}$. The transpeptidation reaction involves three steps. First, the sorting signal is recognized and noncovalently binds to the sortase enzymes. Second, an acylation step occurs during which the active site cysteine performs a nucleophilic attack on the threonine's carbonyl carbon and a semi-stable thioacyl intermediate is formed between the first substrate of catalysis and the sortase. Finally, a second pilin protein harboring an amino-nucleophile lysine sidechain binds to the sortase, and a deacylation reaction occurs during which a new isopeptide bond is formed between the sorting signal threonine and the lysine side chain in the second pilin protein ${ }^{15,2,3,8}$. A repetition of this process results in the growth of the pilus shaft, which can extend more than a micron from the bacteria ${ }^{3,17}$. The pilus biosynthesis reaction is complex, as many pili contain specialized tip proteins that mediate adhesion, as well as base proteins whose incorporation into the growing shaft terminates assembly by promoting pilus attachment to the cell wall ${ }^{3}$. Moreover, bacteria 
frequently display more than one type of pilus, each of which are expressed on gene clusters which can encode two or three pilin proteins and one to three sortases. The molecular basis through which these sortase enzymes function to non-redundantly assemble pili remains largely unknown.

Class $\mathrm{C}$ sortases are unique among the sortase superfamily in that they contain an $\mathrm{N}$-terminal extension known as a "lid" that completely occludes the active site ${ }^{15,18-20}$ (Fig. 1). This lid has been found in all class $\mathrm{C}$ sortases studied to date and invariably maintains a conserved $\mathrm{DP}(\mathrm{F} / \mathrm{W} / \mathrm{Y})$ anchor motif wherein the aspartate residue forms a hydrogen bond with the active site arginine, and the aromatic residue at the end of the motif is wedged into the active site where it forms a sulfur-aromatic interaction with the active site cysteine ${ }^{20}$. Based on the fact that the lid blocks access to the sorting signal binding site seen in all structures reported to date ${ }^{21-23}$, and that several class $\mathrm{C}$ sortase crystal structures are either missing electron density ${ }^{24-27}$ or have elevated B-factors throughout this region ${ }^{18-20}$, it has been proposed that the lid region of these sortases must be highly flexible in solution (see Table S1 for a comparison of these structures) $)^{15,18-20,26,28}$. However, to the best of our knowledge, the flexibility of the lid in solution has never been directly investigated using computational or experimental methods.

The class C sortases of Streptococcus pneumoniae are of particular biological and pharmaceutical interest. $S$. pneumoniae is one of the most common causative agents of multiple diseases, including bacterial pneumonia, sepsis, and meningitis ${ }^{29-31}$, and while not all serotypes rely on pili, pneumococci clones that are nonsusceptible to penicillin have an increased reliance on these structures for their virulence ${ }^{10,32}$. S. pneumoniae can express two distinct types of pili carried on separate genomic islands, known as pilus island 1 (PI-1) or pilus island 2 (PI-2) $)^{33}$. PI-1 is present in $\sim 30 \%$ of $S$. pneumoniae strains, and these pili have been more thoroughly characterized. They consist of three pilus proteins, $\operatorname{Rrg} \mathrm{A}, \operatorname{RrgB}$, and $\mathrm{RrgC}$, which are polymerized by three sortases, SpnSrtC1, SpnSrtC2, and SpnSrtC3. Structures for each of these sortases have been solved, and they are all nearly identical and show that the conserved lid occludes the active site ${ }^{18,24}$. Mutations to the anchor residues in the lid of SpnSrtC1 have been shown to decrease the thermal stability of the enzyme in vitro $^{19}$, as well as alter the pattern of, but not abrogate, the production of polymerized $\operatorname{RrgB}$ species in vitro ${ }^{18}$.

Here, we use experimental and computational techniques to show that despite the generally accepted hypothesis that sortase lids are flexible in solution, the lid of $S$. pneumoniae SrtC1 (hereafter referred to as $\mathrm{SrtC} 1$ ) adopts a rigid structure in vitro that maintains the enzyme in an inactive state. Mutations to the anchor residues of the lid increase its flexibility and dramatically increase the rate of in vitro hydrolysis of a sorting signal, as well as the susceptibility of the active site cysteine to oxidation. Simulation and NMR results provide insight into the mechanism of lid opening, and reveal two dynamic flanking regions that may function as hinges. Our results substantiate the proposed negative regulatory function of the lid in pilus biogenesis and further suggest that additional factors associated with the microbial surface are likely needed to activate the enzyme by dislodging its structurally rigid lid. 


\section{Methods}

\section{Experimental Procedures}

Cloning, protein expression and purification-DNA encoding codon optimized soluble SrtC1, residues 17-228 based on uniprot sequence Q97SB9, was generated by recursive PCR from overlapping primers ${ }^{34}$. D58A, W60A, D58A-W60A double mutant, and GSlid mutants of $\mathrm{SrtC} 1$ were generated by site directed mutagenesis using standard procedures. ${ }^{15} \mathrm{~N}$ and ${ }^{13} \mathrm{C}$ labeled variants were produced by expressing the proteins in M9minimal media supplemented with ${ }^{15} \mathrm{~N} \mathrm{NH}_{4} \mathrm{Cl}$ and/or ${ }^{13} \mathrm{C}$ glucose. Proteins were expressed and purified by IMAC using HisPure $\mathrm{Co}^{2+}$ resin (Thermo) per manufacturer's instructions.

NMR spectroscopy-All protein samples used for NMR experiments were concentrated and dialyzed into NMR buffer ( $50 \mathrm{mM}$ sodium phosphate $\mathrm{pH} 6.5,50 \mathrm{mM} \mathrm{NaCl}, 10 \% \mathrm{D}_{2} \mathrm{O}$ ). NMR spectra were recorded on Bruker 500, 600, and 800 MHZ spectrometers equipped with triple resonance cryogenic probes and processed using NMRPipe ${ }^{35}$. Chemical shift assignments were obtained through the analysis of HNCO, HN(CA)CO, HNCA, HNCACB, $\mathrm{CBCA}(\mathrm{CO}) \mathrm{NH}, \mathrm{CC}(\mathrm{CO}) \mathrm{NH}$, and HBHANH experiments in CARA $1.9 .0^{36} .94 \%$ of backbone resonances could be definitively assigned using standard triple resonance assignment methods, leaving a few one to two residue unassigned segments, and a single seven residue stretch of amino acids (residues Q167-E172) which could not be assigned. An analysis of chemical shifts was used to define the location of the secondary structural elements, which were found to be similar to those present in the previously reported crystal structure. Dilution experiments to investigate the potential for $\mathrm{SrtC} 1$ to dimerize were performed by collecting ${ }^{15} \mathrm{~N}-\mathrm{HSQC}$ spectra beginning at $2 \mathrm{mM}$, and diluting the protein serially with NMR buffer $+10 \% \mathrm{D}_{2} \mathrm{O}$ to $1.5,1,1.5,0.5,0.25,0.2$, and $0.1 \mathrm{mM}$. Dynamics experiments were conducted on $1 \mathrm{mM}{ }^{15} \mathrm{~N}$ labeled samples in NMR buffer described above. Heteronuclear NOE, $\mathrm{T}_{1}$ and $\mathrm{T}_{2}$ experiments were conducted at $298 \mathrm{~K}$ and analyzed in SPARKY ${ }^{37}$. The assigned backbone chemical shifts SrtC1 have been deposited in the Biological Magnetic Resonance Bank (BMRB ID 26745).

In vitro cleavage assay- $\mathrm{SrtC} 1$ and mutants were dialyzed into assay buffer $(25 \mathrm{mM}$ TRIS-HCl pH 7.5, $100 \mathrm{mM} \mathrm{NaCl}$ ). Sorting signal containing peptide: $\mathrm{NH}_{2^{-}}$ VNKKITIPQTGGIGT-CO 2 was synthesized by LifeTein and used without further purification. Lyophilized peptide was dissolved in assay buffer to $2 \mathrm{mM}$ and the final concentration was verified using a BCA assay kit (Thermo) per the manufacturer's instructions. Reactions were conducted with $100 \mu \mathrm{M}$ enzyme and $200 \mu \mathrm{M}$ peptide in a total volume of $100 \mu \mathrm{L}$ at $37^{\circ} \mathrm{C}$. After $24 \mathrm{hrs}$, reactions were quenched by addition of $50 \mu \mathrm{L} 1 \mathrm{M}$ $\mathrm{HCl}$ and $100 \mu \mathrm{L}$ of the reaction mixture was injected onto a Waters XSelect HSS $\mathrm{C}_{18}$ reversed phase HPLC column. Peptides were eluted with a gradient from 5-30\% acetonitrile, $0.1 \%$ trifluoroacetic acid over 20 mins. Elution and quantification of peptides was conducted by monitoring absorbance of the peptide bond at $215 \mathrm{~nm}$. Fractions from peaks corresponding to substrate and product were collected and their identities confirmed by matrix assisted laser desorption ionization-mass spectrometry (MALDI-MS). 


\section{Molecular Dynamics Simulations}

Conventional Molecular Dynamics (cMD) Calculations-The initial coordinates for $\mathrm{SrtC} 1$ were obtained from the protein data bank (PDB) $2 \mathrm{~W} 1 \mathrm{~J}$ structure ${ }^{18}$. Hydrogen atoms were added using the xLeap module of the AMBER software suite ${ }^{38}$, followed by solvation with a box of TIP3 $\mathrm{P}^{39}$ water molecules such that there was a minimum distance of $10 \AA$ between the protein atoms and the surface of the box. $\mathrm{Na}^{+}$and $\mathrm{Cl}^{-}$ions were then added at random positions in the box to neutralize the system and bring the salt concentration to 150 $\mathrm{mM}$, resulting in an orthorhombic box containing approximately 30,000 atoms. The same preparatory steps were applied to create models of the W60A, D58A, D58A/W60A, GSlid and D58G/W60G mutants. The AMBER ff14SB force field ${ }^{40}$ parameters were used for all protein models. Subsequently, two stages of minimization, NVT and NPT equilibrations were carried out to prepare the systems for production simulations. Time steps of 1.0 and 2.0 fs were used during the equilibration and production stages respectively. The SHAKE algorithm ${ }^{41}$ was used to fix bonds involving hydrogens to their equilibrium values. A $10 \AA$ cutoff was used for van der Waals interactions, while long range electrostatic interactions were modeled with particle mesh Ewald (PME) summation ${ }^{42}$ using a $1.0 \AA$ grid $^{6-7}$. The PMEMD module of the AMBER software suite with GPU acceleration was used to carry out the MD calculations ${ }^{43,44}$.

\section{Replica Exchange Molecular Dynamics (REMD) Calculations-REMD} calculations ${ }^{45}$ were implemented using 32 replicas with temperature ranging from 300 to $380 \mathrm{~K}$ that were distributed using the method developed by van der Spoel and coworkers ${ }^{46}$. Exchange attempts were made every $125 \mathrm{MD}$ steps while all other simulation parameters were the same as the cMD calculations. A 200 ns per replica calculation was carried out for $\mathrm{SrtC1}$ and D58G/W60G, leading to a total simulation time of $6.4 \mu$ s per protein.

Umbrella Sampling Calculations-Umbrella sampling calculations were implemented to quantify the energetics of lid opening with a reaction coordinate defined as a collective variable based on a full correlation analysis ${ }^{47}$ (FCA) of the REMD trajectories of the WT and D58G/W60G mutant ${ }^{47}$. The first FCA mode, which described the lid opening process, was sampled between $-10.0 \AA$ and $28.0 \AA$ using 20 windows and a $2.0 \mathrm{kcal} / \mathrm{mol} \AA^{2}$ force constant. A $100 \mathrm{~ns}$ calculation was implemented per window for each system, resulting in $2.0 \mu$ s total sampling per protein with the first $40 \mathrm{~ns}$ of each window discarded as equilibration. The weighed histogram analysis method (WHAM), as implemented in the program by Dr. Grossfield ${ }^{48}$, was applied to obtain the potential of mean force (PMF) of lid opening. The colvar module ${ }^{49}$ of the NAMD ${ }^{50}$ software was used for all umbrella sampling simulations with the same force field and simulation parameters as was used for $\mathrm{cMD}$ calculations in AMBER.

\section{Results}

\section{NMR and MD results indicate that the SrtC1 lid is not dynamic in vitro}

NMR Measurements-NMR experiments were conducted to determine whether the lid of $\mathrm{SrtC} 1$ is mobile in solution. Initially, ${ }^{15} \mathrm{~N} \mathrm{~T}_{1}, \mathrm{~T}_{2}$, and HetNOE experiments were performed to measure the relaxation properties of backbone amide nitrogens on ps-ns timescales. $\mathrm{T}_{1}$ 
and $\mathrm{T}_{2}$ results were used to estimate the rotational correlation time, $\tau_{\mathrm{e}}$, for each backbone amide residue in the protein (Fig. 2). Residues that sample disordered states are expected to have a $\tau_{\mathrm{e}}$ value lower than the protein's overall rotational correlation time, $\tau_{\mathrm{c}}$, (Fig.2A, red line) since they can move through space more rapidly than the protein body. In the crystal structure of $\mathrm{SrtC1}$, the lid spans residues 50-72 and interacts with the active site via the anchor residues D58 and W60. The lid also contains a short helix formed by residues 61-66 that packs against helix H6 that is positioned adjacent to the active site. Surprisingly, lid residues D58 and W60, which contact the active site, have $\tau_{\mathrm{e}}$ values of $16.4 \mathrm{~ns}$ and $16.9 \mathrm{~ns}$ respectively, which is similar to the estimated value of $\tau_{c}=17.8 \pm 1.1 \mathrm{~ns}$ for the protein core, whereas regions immediately before and after these residues have reduced $\tau_{\mathrm{e}}$ values. This is in agreement with the HetNOE data collecte $\mathrm{d}$ at a ${ }^{15} \mathrm{~N}$ field strength of $60.8 \mathrm{MHz}$, for which values above 0.6 indicate residues that are structured in solution, while those with values below 0.6 are considered disordered (Fig. 2B, red line). Notably, residues 56-57 and 65-70, which are located immediately before and after the portion of the lid that contacts the active site, show increased motions on this timescale, indicating that they have elevated mobility. In contrast, the lid residues between these segments (residues 58-64) that contain the active site contacting D58 and W60 residues have HetNOE values above 0.6 and $\tau_{\mathrm{e}}$ values similar to residues involved in regular secondary structure elements, indicating that they adopt a more rigid state.

Additional experiments were conducted to determine if the lid exhibits large amplitude motions that occur more slowly on the $\mu \mathrm{s}-\mathrm{ms}$ timescale. To measure this phenomenon, we first conducted CPMG experiments ${ }^{51}$ to directly detect the $\mathrm{R}_{\mathrm{ex}}$ component of relaxation on a per residue basis (Fig. 2C). $R_{\mathrm{ex}}$ values are generally considered to be an indicator of slower timescale motions if they are greater than $\sim 30 \%$ of the measured $\mathrm{T}_{2}$ for an individual residue, which typically means values above $10 \mathrm{~s}^{-1}$ are considered significant ${ }^{52}$. Residues in and around the lid region did not show significantly elevated $R_{\mathrm{ex}}$ values, indicating that they are not mobile on the $\mu$ s-ms timescale. Moreover, the results of chemical exchange saturation transfer (CEST) experiments did not reveal the presence of "invisible" excited, less populated protein state(s) that are in slow exchange with a more abundant ground-state conformation (data not shown) ${ }^{52}$. This indicates that the lid is likely closed in $>99 \%$ of the protein in solution, given that CPMG and CEST experiments are capable of detecting excited states with fractional populations as low as $0.5 \%$ of the total protein in solution ${ }^{52}$. Collectively, the NMR data and crystal structure indicate that the lid region adopts a closed and rigid state, and that it is the predominant form of the apo-enzyme enzyme in solution. However, residues immediately preceding and following the anchoring residues in lid have slightly elevated mobility suggesting that they may potentially function as hinge points that undergo motions that dislodge the lid from the active site.

Molecular Modeling-SrtC lid dynamics were further characterized through a series of molecular modeling calculations. Initially, a $1.0 \mu$ s conventional molecular dynamics (cMD) simulation was performed, and both the root mean square deviations (RMSD) of heavy atoms from the crystal structure, and root mean squared fluctuations (RMSF) of the $\mathrm{C}_{a}$ atoms were computed to quantify the dynamics (Fig. 3). 
Over the course of the simulation, the protein remained structurally close to the starting Xray structure, with a maximum RMSD of $\sim 2.7 \AA$. Additionally, the D58-R202 distance, which measures contact between the anchor residue in the lid and the active site, remained at $4.0 \pm 0.1 \AA$ for the duration of the $1.0 \mu \mathrm{s} \mathrm{cMD} \mathrm{calculation} \mathrm{(Fig.} 3 \mathrm{C}$ ) indicating a strong salt bridge between both residues. Furthermore, W60 and C193 stayed in close proximity to one another (W60-C193 = 4.1 $\pm 0.5 \AA$ ), allowing for a T-shaped interaction for most of the cMD calculation (Fig. 3D). However, slightly higher RMSF values were calculated for the regions around the anchoring residues D58 and W60, which were experimentally observed to be more dynamic based on their decreased HetNOEs and $\tau_{\mathrm{e}}$ values (Fig. 2). High RMSF values were also observed for a section of the $\beta 5 / \beta 6$ loop (residues 134 to 149 ) which showed slightly elevated B-factors in the $2 \mathrm{~W} 1 \mathrm{~J}$ crystal structure, although this was not corroborated by our NMR data.

The cMD calculations revealed that several portions of SrtC are flexible, including portions of the lid region. However, large-scale dynamics, such as a hypothetical lid opening motion, may not occur over the course of a $1.0 \mu \mathrm{s} \mathrm{cMD}$ simulation. Therefore, replica exchange molecular dynamics (REMD) calculations were performed to enhance sampling beyond the $\mu$ s-timescale. In REMD, several copies of the same system are concurrently simulated at different temperatures to facilitate the crossing of energy barriers ${ }^{45}$. REMD calculations covering temperatures from 300 to $380 \mathrm{~K}$ were performed and analyzed in the same manner as the cMD simulations above (see SI for details). Overall, REMD results were similar to those from cMD simulations. In particular, the D58-R202 salt-bridge and W60-C193 separation distances were $4.0 \pm 0.2 \AA$ and $4.5 \pm 0.6 \AA$ respectively, suggesting that interactions that anchor the lid into the active site are robust over long-timescales. Taken together, our NMR and MD experiments indicate that the SrtC1 lid is not dynamic in solution, as has been previously suggested, and is instead stabilized by strong lid/active site interactions that keep it largely structured in the absence of additional factors, even out to very long timescales.

\section{Mutations in the lid increase its flexibility}

It has previously been shown that the thermostability of $\mathrm{SrtC} 1$ is lowered by mutations to the conserved Asp and Trp residues in the DP(F/W/Y) lid motif ${ }^{19}$. In addition, analogous mutations in the lid of the Streptococcus agalactiae sortase C-1 increase the rate at which it catalyzes pilin polymerization and sorting signal hydrolysis ${ }^{53,54}$. These results suggest that mutations to the $\mathrm{SrtC} 1$ lid may destabilize its structure and thereby promote substrate access to the active site. To investigate this, NMR and computational methods were used to study the effects of D58A and W60A mutations on SrtC1 lid dynamics.

As compared to the wild-type enzyme, the lid in the W60A mutant has significantly increased mobility. Experimentally derived $\tau_{\mathrm{e}}$ values indicate that this region tumbles at 10.7 ns, $5.2 \mathrm{~ns}$ faster than the average for the structured portion of this enzyme (Fig. 4). HetNOE data confirms that these residues are largely disordered; every residue that could be assigned and characterized between the two potential hinge points (residues 58-64) had reduced HetNOE values compared to WT, with many having values below 0.6. cMD simulations on the W60A mutant also showed an increase in dynamics relative to the WT enzyme. As with 
the WT enzyme, CPMG and CEST experiments performed with the W60A mutant showed no evidence of longer timescale dynamics that would indicate the presence of slower concerted motions associated with lid opening (data not shown). Furthermore, for all mutants, lower hetNOE values are observed, consistent with the lid in these proteins undergoing increased motions on the pico-second timescale. In particular, the average RMSF values for the anchoring residues 58-60 were measured to be $1.5 \AA$, which is higher than the value of $0.7 \AA$ that was observed for the WT (Fig. 5). Additionally, W60A had RMSD values for the lid region of $\sim 4.5 \AA$ relative to the X-ray structure, while the WT had an overall lid RMSD of $\sim 1.0 \AA$ (Fig. 6).

NMR and simulation data also show that the lid in the SrtC1-D58A mutant has significantly increased lid mobility as compared to the wild-type protein (Figs. 4-6). Examination of the cMD trajectory of D58A showed that the W60 moiety moves from its crystal structure position and docks into a hydrophobic nook in the active site groove where it is surrounded by residues V36, L93, F157 and L160 (Fig. S2). The unique molecular environment experienced by W60 in this mutant is illustrated by the contact histograms for the D58A and WT proteins, which shows the total contact fractions between W60 sidechain atoms and neighboring residues (Fig. 7). Notably, in the D58A mutant, W60 is no longer in regular contact with the catalytic cysteine but is in more frequent contact with R202 due to the broken salt bridge interaction. This finding is compatible with the NOESY data of the D58A mutant, which reports on the local environment of the W60 indole proton. In the $3 \mathrm{D}{ }^{1} \mathrm{H}_{-}{ }^{15} \mathrm{~N}$ NOESY HSQC spectrum of the wild-type protein, numerous cross peaks are observed to this atom, consistent with the W60 side chain residing within the active site of the enzyme as observed in the crystal structure (in the NOESY spectrum, cross-peaks to the indole proton occur if it is within $\sim 5 \AA$ of another proton). In contrast, in the NOESY spectrum of the D58A mutant, only intra-residue NOE cross peaks are observed, suggesting that the indole ring does not adopt a stable state within the active site. This is further supported by the ${ }^{1} \mathrm{H}_{-}{ }^{15} \mathrm{~N}$ HSQC spectrum of the D58A protein, as several cross-peaks in the spectrum that presumably correspond to the indole proton are present, all of which exhibit narrower linewidths than the indole signal in the spectrum of the wild-type protein. Together, both the experimental and computational results demonstrate that while portions of the lid in the D58A mutant appear as rigid as the WT enzyme, the conformation of this entire region has been altered significantly, and the native, closed state of the lid has been disrupted.

More substantial alterations in the lid were investigated using cMD simulations. These included both a D58A/W60A and D58G/W60G double mutant proteins, as well as a mutant where residues 55-69 of the lid were replaced with a 15 residue glycine-serine linker (SrtC1-GSlid). Each mutant showed an increase in lid fluctuations relative to the wild-type. In particular, RMSF values were higher for these mutants than the WT, particularly for the anchor residues (Fig. 5). The D58G/W60G and GSlid mutants had the most mobile lids, with RMSF values over $5.0 \AA$ and lid RMSDs over $6.0 \AA$ for both systems (Fig. 6 and S3). Examination of these simulations showed that the lid adopted an open state with the active site groove exposed to solution (Fig. 8). Meanwhile, the D58A/W60A simulation exhibited dynamics that were similar to the previously discussed W60A and D58A single mutants, with the lid having increased dynamics to the WT, but not fully opening as in the D58G/ W60G and GSlid systems. 


\section{Mutations in the lid increase enzyme activity and substrate access}

Having rigorously established that $\mathrm{SrtC} 1$ lid mutations increase its mobility, we set out to determine whether these alterations also facilitated sorting signal binding and catalysis as might be expected if the native lid negatively regulates enzyme activity. Previous studies have reported that $\mathrm{SrtC} 1$ can polymerize $\mathrm{RrgB}$ in vitro ${ }^{18,19}$. However, for unknown reasons, our repeated attempts to reproduce this activity proved unsuccessful (data not shown). We therefore tested SrtCl's activity with an in vitro hydrolysis assay that monitored the ability of the enzyme to cleave a 15 amino acid peptide that is derived from the $\operatorname{RrgB}$ protein and contains the IPQTG sorting signal recognized by this sortase. This assay reports on the first step of pilin assembly: nucleophilic attack of the sortase cysteine thiol on the Thr carbonyl carbon atom within the IPQTG motif. In the assay, the enzyme and peptide are incubated for $24 \mathrm{hrs}$ at $37^{\circ} \mathrm{C}$, and then the reaction components are separated by HPLC. This enables the progress of the reaction to be determined by monitoring both the amount of cleaved product peptide formed, as well as the disappearance of the substrate peptide. The assay was used to measure the activities of wild type SrtC1, and proteins containing D58A, W60A, C193A, D58A/W60A and GSlid mutations. The GSlid construct was used as an approximation of a completely open lid, since $\mathrm{SrtC} 1$ mutants that removed the entire $\mathrm{N}$-terminal extension that contains the lid aggregated in solution and could not be assayed.

The assay data shown in Figure 9 demonstrates that lid mutations that destabilize the lid structure increase the enzyme's ability to cleave the sorting signal. No detectable product was observed when the wild-type enzyme was incubated with IPQTG containing peptide (Fig. 9). As expected, a negative control C193A mutant which lacks the catalytic cysteine was also inactive. In contrast, sortase enzymes containing mutations that destabilize lid contacts to the active site were enzymatically active and catalyzed peptide hydrolysis. The activity of all of the mutants was nearly identical (within the error of the measurement), except for the D58A mutant, which was clearly less active ( $p<0.01$ vs W60A or W60AD58A mutants, $p<0.05$ vs SrtC1-GSlid). The lower activity of D58A could be due to the partial occlusion of the active site by the lid, which is compatible with the results of MD simulations that indicate that the lid in D58A is less mobile than in the W60A mutant (Fig. $4)$.

The elevated hydrolytic activity of lid mutant proteins suggests that the lid in the wild-type enzyme may negatively regulate enzymatic activity by obstructing the binding site for the sorting signal. To investigate this, we performed disulfide bonding experiments utilizing a modified Cbz-IPQT* sorting signal substrate, where $\mathrm{Cbz}$ is a carbobenzyloxy protecting group and $\mathrm{T}^{*}$ is a modified thiol containing threonine residue $((2 R, 3 S)$-3-amino-4mercapto-2-butanol) that can form a disulfide bond with the active site cysteine. In other sortases, this type of peptide analog has been successfully used to produce stable enzymesubstrate complexes ${ }^{21-23}$. When incubated with the IPQT* substrate at room temperature for $24 \mathrm{hrs}$, the WT SrtC1 showed no detectable disulfide formation by MALDI-MS (Fig. S4). When the same experiment was repeated with the W60A mutant, a mass shift of $595 \mathrm{Da}$ (theoretical mass of Cbz-IPQT* $* 593.4 \mathrm{Da}$ ) was detected, indicating the formation of a disulfide-bonded complex. The difference in cysteine availability highlighted by the results 
of this experiment indicate that the lid in WT SrtC1 physically limits sorting signal access to the active site cysteine.

\section{The thermodynamics of lid opening correlate with SrtC1 catalytic activity}

In light of the experimental and computational findings described above, umbrella sampling calculations were performed to quantify the effects of mutations on the free energy of lid opening. The reaction coordinate for these simulations was defined by a full correlation analysis (FCA) of $\mathrm{C}_{a}$ atoms in the WT and D58G/W60G REMD calculations (see Methods and SI for details). Specifically, a collective variable was defined based upon a projection of the system on the first FCA mode (FCA 1). This reaction coordinate primarily describes the conformational transition between a closed and an open lid, with projections around $-6 \AA$ corresponding to a closed state, and values above $15 \AA$ to an open state (see Movie S1).

Projections of the WT, GSlid, and all alanine mutants cMD trajectories on FCA1 revealed similar dynamical information as outlined above (Fig. S5), and demonstrate that this coordinate can reliably measure lid opening. For example, the WT mostly samples trajectories with FCA 1 values of -6 A (closed states), while the GSlid mutant evolves over time from a closed to an open (FCA $1>15 \AA$ ) conformation. As was observed in the RMSD and RMSF comparisons, the W60A, D58A and D58A/W60A were intermediate between the WT and the GSlid, indicating they preferentially sample conformations between the open and closed states.

The potentials of mean force (PMFs) demonstrate that the WT lid samples only the closed state, and that lid mutations significantly reduce the free energy cost of lid opening (Fig. 10). The WT PMF has a single minimum in the region of $-6 \AA$, whereas conformations with an FCA 1 projection $>15 \AA$ have free energies on the order of $20 \mathrm{kcal} / \mathrm{mol}$. In contrast, and in line with the MD data described above, the open conformations of the GSlid mutant were easily accessible, requiring less than $3 \mathrm{kcal} / \mathrm{mol}$ of energy to sample states with FCA $1>15$ $\AA$. Opening of the D58A and D58A/W60A mutants required considerably less free energy than the WT (under $10 \mathrm{kcal} / \mathrm{mol}$ for FCA $1 \sim 15 \AA$ ), while the W60A mutant required slightly higher energies at $\sim 12 \mathrm{kcal} / \mathrm{mol}$. In addition to modifying the free energies of the closed and open lid states, mutants affect the thermodynamics of intermediate "semi-open" conformations. For D58A/W60A and W60A, the PMFs show minima at around 2-3 A, with access to FCA $1 \sim 6 \AA$ (partially open lid) requiring on the order of $1.5 \mathrm{kcal} / \mathrm{mol}$ of free energy. The minimum for D58A is also located at a value corresponding to a more open structure $(\sim-1.0 \AA)$ compared to the WT.

\section{Discussion and Conclusion}

The ability of class $\mathrm{C}$ sortases to catalyze the covalent linkage between protein substrates, instead of attaching target proteins to the cell wall, makes them unique among the sortase family of enzymes ${ }^{15}$. Class $\mathrm{C}$ sortases are structurally unique in that they contain an $\mathrm{N}$ terminal polypeptide segment that precedes the catalytic domain. This extension projects into the active site and has been termed the $\mathrm{lid}^{15,18-20}$. It has been generally thought that the lid structure is flexible and thus capable of transiently being displaced from the active site. This hypothesis was based on several crystal structures of class $\mathrm{C}$ enzymes that frequently 
have elevated B-factors or are missing electron density for the backbone atoms in portions of the lid. In addition, multiple studies have shown that proteins in which the lid is removed or mutated have elevated enzymatic activity, suggesting that the lid may negatively regulate activity when it interacts with the enzyme's active site and that lid removal is necessary for substrates to access the active site $15,18-20,26$. However, the dynamics of the lid, and how it participates in substrate binding, had not been directly determined.

Here, we systematically investigated the dynamics and function of the lid in the $S$. pneumonia $\mathrm{SrtC} 1$ protein using biochemical, NMR, and MD methods. Surprisingly, our NMR results indicate that in the wild-type enzyme, residues in the lid that contact the active site adopt stable, largely immobile conformations. In this rigid state, the enzyme is inactive and incapable of interacting with its sorting signal substrate. Conventional MD and REMD calculations of the wild-type confirm the rigid structure of the lid, which is held tightly over the active site by both a salt-bridge between D58 and R202, as well as an aromatic-sulfur interaction between W60 and C193. Umbrella sampling calculations show that the free energy of lid opening is prohibitive in the WT enzyme, and that on its own SrtC1 will not sample states with an open lid. In addition, both NMR and computational results support the hypothesis that the N-terminal extension contains "hinges," an idea that was originally proposed by Manzano and coworkers based on elevated B-factors in the SrtC3 crystal structure for the two discrete regions that flank the lid $^{18}$. This hypothesis is further supported by the significant number of $\mathrm{SrtC}$ structures solved to date wherein these regions either showcase the highest B-factors in the structure, or lack density altogether ${ }^{18-20,24-27}$.

Our results indicate that mutations which increase lid flexibility, improve its ability to interact with the sorting signal and to perform the first step of catalysis. For a series of lid mutants, we characterized the dynamic properties, sorting signal accessibility, and the catalytic activity of the enzyme to perform the initial cleavage step in transpeptidation reaction in vitro. Only when mutations to the lid are introduced which increase lid flexibility, as indicated by reduction in both the $\tau_{\mathrm{e}}$ and HetNOE and an increase in RMSD and RMSF values for these residues (Figs. 4 and 5), does the enzyme catalyze the initial reaction step in vitro (Fig. 9). The same motion-inducing mutations are also required to grant access to the cysteine for the production of a disulfide bond, which would not require access to the rest of the binding site or participation from other active site residues (Fig. S4). This indicates that rigidity of the WT lid prevents hydrolysis of the enzyme's native substrate in solution and does so by completely preventing access to the active site cysteine instead of simply inactivating the enzyme via conformational manipulation or obstruction of individual recognition pockets in the binding site.

The mechanisms through which the lid may be dislodged from the active site were investigated using MD simulations. The data shows that breaking the D58-R202 and W60AC193 interactions via mutations to either alanine or glycine residues allows the lid region to adopt a variety of conformational states, many of which are open. Completely open lid structures resemble the conformation shown in Fig. 7 with the lid region rolled back from the active site. The mutants with more extensive mutations, such as the GSlid, transition to majority open states, while single mutants such as D58A have partially open lids. These motions are compatible with the NMR relaxation data, as the lid opens at residues 56-57 
and 60-65. These sites function as hinges for lid displacement and exhibited slightly elevated mobility in the wild-type enzyme, suggesting that they are primed to undergo conformational change. In addition, free energy calculations quantitatively demonstrate that mutant proteins access conformations with the lid open more frequently than the wild-type (Figs. 5-6, 10). This finding correlates with the lack of in vitro hydrolysis observed experimentally for the WT compared to the increased reactivity shown for the mutants. Furthermore, the fact that mutants with free energy minima corresponding to semi-open states (such as W60A and D58A/W60A) are as catalytically competent as mutants that prefer an open lid state (such as the GSlid) suggests that it may not be necessary for a mutant to sample the fully open state in vitro to perform catalysis. This hypothesis is further supported by the fact that the D58A mutant is less active in vitro then the W60A mutant despite it having a lower free energy penalty for sampling a fully open lid state. Taken together, this data suggests that the semi-open states may sufficiently weaken the D58-R202 and W60-C193 interactions to facilitate lid opening and by extension catalysis.

The available data are compatible with the lid in $\mathrm{SrtC} 1$ serving a regulatory role in which it holds the enzyme in an inactive state on the cell surface until interactions with other protein factors and/or the membrane initiate pilus biogenesis by prying the lid from the enzyme. It is advantageous for the lid to hold the enzyme in a dormant state until all of the appropriate factors are present, as pilus assembly is an energetic costly process that involves the covalent linkage of $\sim 100$ protein subunits. Because SrtC1 and other sortases hydrolyze their sorting signal substrates when the secondary nucleophile is absent, holding the enzyme in a dormant state would be particularly useful as it could prevent the spurious, hydrolytic release of incompletely assembled pili. It is also possible that the main function of the lid is to prevent the oxidation of the active site cysteine residue that is required for catalysis. The active site cysteine residues in other sortase enzymes have been shown to be inactivated by oxidation $^{55,56}$, and it has been proposed that during infections hosts cells may create an oxidizing environment to deactivate sortases and thereby render the bacterial pathogen less virulent ${ }^{56,57}$. The fact that we observed rapid formation of a disulfide linked complex between a sorting signal mimic and the W60A lid mutant of SrtC1, but not between the same mimic and the WT protein, suggests that the lid indeed functions to prevent oxidation of the active site cysteine (Fig. 9). As bacteria expressing SrtC1 lid mutants are still capable of assembling pili in cell culture, a proper functioning lid is not essential, and most likely confers a fitness advantage to the microbe by enabling it to avoid energetically wasteful and spurious pili release and/or sortase inactivation ${ }^{53,58}$. A greater understanding of the function of the lid and the mechanism of pilus biogenesis will require the structure determination and computational modeling of intact reaction intermediates and could lead to the development of new therapeutics that work by disrupting pilus display.

\section{Supplementary Material}

Refer to Web version on PubMed Central for supplementary material.

\section{Acknowledgments}

This work was supported, in whole or in part, by National Institutes of Health Grants K22AI104799 (to J.W.) and AI52217 (to R.T.C), as well as funding from the US Department of Energy Office of Science, Office of Biological 
and Environmental Research program under Award Number DE-FC02-02ER63421.This work used the Extreme Science and Engineering Discovery Environment (XSEDE), which is supported by National Science Foundation Grant No. ACI-1053575.

\section{References}

1. Ton-That H, Schneewind O. Assembly of Pili in Gram-Positive Bacteria. Trends Microbiol. 2004; 12:228-234. [PubMed: 15120142]

2. Telford JL, Barocchi MA, Margarit I, Rappuoli R, Grandi G. Pili in Gram-Positive Pathogens. Nat. Rev. Microbiol. 2006; 4:509-519. [PubMed: 16778837]

3. Mandlik A, Swierczynski A, Das A, Ton-That H. Pili in Gram-Positive Bacteria: Assembly, Involvement in Colonization and Biofilm Development. Trends Microbiol. 2008; 16:33-40. [PubMed: 18083568]

4. Rintahaka J, Yu X, Kant R, Palva A, von Ossowski I. Phenotypical Analysis of the Lactobacillus Rhamnosus GG Fimbrial spaFED Operon: Surface Expression and Functional Characterization of Recombinant SpaFED Pili in Lactococcus Lactis. PloS One. 2014; 9:e113922. [PubMed: 25415357]

5. Mishra A, Wu C, Yang J, Cisar JO, Das A, Ton-That H. The Actinomyces Oris Type 2 Fimbrial Shaft FimA Mediates Co-Aggregation with Oral Streptococci, Adherence to Red Blood Cells and Biofilm Development. Mol. Microbiol. 2010; 77:841-854. [PubMed: 20545853]

6. Mishra A, Devarajan B, Reardon ME, Dwivedi P, Krishnan V, Cisar JO, Das A, Narayana SVL, Ton-That H. Two Autonomous Structural Modules in the Fimbrial Shaft Adhesin FimA Mediate Actinomyces Interactions with Streptococci and Host Cells during Oral Biofilm Development. Mol. Microbiol. 2011; 81:1205-1220. [PubMed: 21696465]

7. Margarit I, Rinaudo CD, Galeotti CL, Maione D, Ghezzo C, Buttazzoni E, Rosini R, Runci Y, Mora M, Buccato S, et al. Preventing Bacterial Infections with Pilus-Based Vaccines: The Group B Streptococcus Paradigm. J. Infect. Dis. 2009; 199:108-115. [PubMed: 19086816]

8. Hendrickx APA, Budzik JM, Oh S-Y, Schneewind O. Architects at the Bacterial Surface - Sortases and the Assembly of Pili with Isopeptide Bonds. Nat. Rev. Microbiol. 2011; 9:166-176. [PubMed: 21326273]

9. Young PG, Moreland NJ, Loh JM, Bell A, Atatoa Carr P, Proft T, Baker EN. Structural Conservation, Variability, and Immunogenicity of the T6 Backbone Pilin of Serotype M6 Streptococcus Pyogenes. Infect. Immun. 2014; 82:2949-2957. [PubMed: 24778112]

10. Barocchi MA. A Pneumococcal Pilus Influences Virulence and Host Inflammatory Responses. Proc. Natl. Acad. Sci. U.S.A. 2006; 103:2857-2862. [PubMed: 16481624]

11. Mandlik A, Swierczynski A, Das A, Ton-That H. Corynebacterium Diphtheriae Employs Specific Minor Pilins to Target Human Pharyngeal Epithelial Cells. Mol. Microbiol. 2007; 64:111-124. [PubMed: 17376076]

12. Maresso AW, Schneewind O. Sortase as a Target of Anti-Infective Therapy. Pharmacol. Rev. 2008; 60:128-141. [PubMed: 18321961]

13. Walsh C. Where Will New Antibiotics Come From? Nat. Rev. Micro. 2003; 1:65-70.

14. Comfort D, Clubb RT. A Comparative Genome Analysis Identifies Distinct Sorting Pathways in Gram-Positive Bacteria. Infect. Immun. 2004; 72:2710-2722. [PubMed: 15102780]

15. Spirig T, Weiner EM, Clubb RT. Sortase Enzymes in Gram-Positive Bacteria. Mol. Microbiol. 2011; 82:1044-1059. [PubMed: 22026821]

16. Jacobitz AW, Wereszczynski J, Yi SW, Amer BR, Huang GL, Nguyen AV, Sawaya MR, Jung ME, McCammon JA, Clubb RT. Structural and Computational Studies of the Staphylococcus Aureus Sortase. J. Biol. Chem. 2014; 289:8891-8902. [PubMed: 24519933]

17. Ton-That H, Schneewind O. Assembly of Pili on the Surface of Corynebacterium Diphtheriae. Mol. Microbiol. 2003; 50:1429-1438. [PubMed: 14622427]

18. Manzano C, Contreras-Martel C, El Mortaji L, Izoré T, Fenel D, Vernet T, Schoehn G, Di Guilmi AM, Dessen A. Sortase-Mediated Pilus Fiber Biogenesis in Streptococcus Pneumoniae. Structure. 2008; 16:1838-1848. [PubMed: 19081060] 
19. Manzano C, Izoré T, Job V, Di Guilmi AM, Dessen A. Sortase Activity Is Controlled by a Flexible Lid in the Pilus Biogenesis Mechanism of Gram-Positive Pathogens. Biochemistry. 2009; 48:10549-10557. [PubMed: 19810750]

20. Persson K. Structure of the Sortase AcSrtC-1 from Actinomyces Oris. Acta Crystallogr. D Biol. Crystallogr. 2011; 67:212-217. [PubMed: 21358052]

21. Jacobitz AW, Wereszczynski J, Yi SW, Amer BR, Huang GL, Nguyen AV, Sawaya MR, Jung ME, McCammon JA, Clubb RT. Structural and Computational Studies of the Staphylococcus Aureus Sortase B-Substrate Complex Reveal a Substrate-Stabilized Oxyanion Hole. J. Biol. Chem. 2014; 289:8891-8902. [PubMed: 24519933]

22. Suree N, Liew CK, Villareal VA, Thieu W, Fadeev EA, Clemens JJ, Jung ME, Clubb RT. The Structure of the Staphylococcus Aureus Sortase-Substrate Complex Reveals How the Universally Conserved LPXTG Sorting Signal Is Recognized. J. Biol. Chem. 2009; 284:24465-24477. [PubMed: 19592495]

23. Chan AH, Yi SW, Terwilliger AL, Maresso AW, Jung ME, Clubb RT. Structure of the Bacillus Anthracis Sortase A Enzyme Bound to Its Sorting Signal: A Flexible Amino-Terminal Appendage Modulates Substrate Access. J. Biol. Chem. 2015; 290:25461-25474. [PubMed: 26324714]

24. Neiers F, Madhurantakam C, Fälker S, Manzano C, Dessen A, Normark S, Henriques-Normark B, Achour A. Two Crystal Structures of Pneumococcal Pilus Sortase C Provide Novel Insights into Catalysis and Substrate Specificity. J. Mol. Biol. 2009; 393:704-716. [PubMed: 19729023]

25. Khare B, Krishnan V, Rajashankar KR, I-Hsiu H, Xin M, Ton-That H, Narayana SV. Structural Differences between the Streptococcus Agalactiae Housekeeping and Pilus-Specific Sortases: SrtA and SrtC1. PLoS ONE. 2011; 6:e22995. [PubMed: 21912586]

26. Khare B, Fu Z-Q, Huang I-H, Ton-That H, Narayana SVL. The Crystal Structure Analysis of Group B Streptococcus Sortase C1: A Model for the "Lid" Movement upon Substrate Binding. J. Mol. Biol. 2011; 414:563-577. [PubMed: 22033482]

27. Cozzi R, Prigozhin D, Rosini R, Abate F, Bottomley MJ, Grandi G, Telford JL, Rinaudo CD, Maione D, Alber T. Structural Basis for Group B Streptococcus Pilus 1 Sortases C Regulation and Specificity. PLoS ONE. 2012; 7:e49048. [PubMed: 23145064]

28. Lu G, Qi J, Gao F, Yan J, Tang J, Gao GF. A Novel “Open-Form” Structure of Sortase C from Streptococcus Suis. Proteins: Struct. Funct. Bioinforma. 2011; 79:2764-2769.

29. Bogaert D, Hermans PWM, Adrian PV, Rümke HC, Groot R de. Pneumococcal Vaccines: An Update on Current Strategies. Vaccine. 2004; 22:2209-2220. [PubMed: 15149779]

30. O'Brien KL, Wolfson LJ, Watt JP, Henkle E, Deloria-Knoll M, McCall N, Lee E, Mulholland K, Levine OS, Cherian T. Burden of Disease Caused by Streptococcus Pneumoniae in Children Younger than 5 Years: Global Estimates. The Lancet. 374:893-902.

31. Levine OS, O’Brien KL, Knoll M, Adegbola RA, Black S, Cherian T, Dagan R, Goldblatt D, Grange A, Greenwood B, et al. Pneumococcal Vaccination in Developing Countries. The Lancet. 367:1880-1882.

32. Sjöström K, Blomberg C, Fernebro J, Dagerhamn J, Morfeldt E, Barocchi MA, Browall S, Moschioni M, Andersson M, Henriques F, et al. Clonal Success of Piliated Penicillin Nonsusceptible Pneumococci. Proc. Natl. Acad. Sci. U.S.A. 2007; 104:12907-12912. [PubMed: 17644611]

33. Danne C, Dramsi S. Pili of Gram-Positive Bacteria: Roles in Host Colonization. Res. Microbiol. 2012; 163:645-658. [PubMed: 23116627]

34. Prodromou C, Pearl LH. Recursive PCR: A Novel Technique for Total Gene Synthesis. Protein. Eng. 1992; 5:827-829. [PubMed: 1287665]

35. Delaglio F, Grzesiek S, Vuister GW, Zhu G, Pfeifer J, Bax A. NMRPipe: A Multidimensional Spectral Processing System Based on UNIX Pipes. J. Biomol. NMR. 1995; 6:277-293. [PubMed: 8520220]

36. Keller, R. Version 1.9.0. Goldau: Cantina Verlag; 2004. CARA: Computer Aided Resonance Assignment.

37. Goddard, TD.; Kneller, DG. Sparky Version 3. San Francisco, CA: Univ. California; 2006. 
38. Case DA, Cheatham TE, Darden T, Gohlke H, Luo R, Merz KM, Onufriev A, Simmerling C, Wang B, Woods RJ. The Amber Biomolecular Simulation Programs. J. Comput. Chem. 2005; 26:16681688. [PubMed: 16200636]

39. Jorgensen WL, Chandrasekhar J, Madura JD, Impey RW, Klein ML. Comparison of Simple Potential Functions for Simulating Liquid Water. J. Chem. Phys. 1983; 79:926-935.

40. Maier JA, Martinez C, Kasavajhala K, Wickstrom L, Hauser KE, Simmerling C. ff14SB: Improving the Accuracy of Protein Side Chain and Backbone Parameters from ff99SB. J. Chem. Theory Comput. 2015; 11:3696-3713. [PubMed: 26574453]

41. Ryckaert J-P, Ciccotti G, Berendsen HJ. Numerical Integration of the Cartesian Equations of Motion of a System with Constraints: Molecular Dynamics of N-Alkanes. J. Comput. Phys. 1977; 23:327-341.

42. Essmann U, Perera L, Berkowitz ML, Darden T, Lee H, Pedersen LG. A Smooth Particle Mesh Ewald Method. J. Chem. Phys. 1995; 103:8577-8593.

43. Götz AW, Williamson MJ, Xu D, Poole D, Grand SL, Walker RC. Routine Microsecond Molecular Dynamics Simulations with AMBER on GPUs. 1. Generalized Born. J. Chem. Theory Comput. 2012; 8:1542-1555. [PubMed: 22582031]

44. Salomon-Ferrer R, Götz AW, Poole D, Grand SL, Walker RC. Routine Microsecond Molecular Dynamics Simulations with AMBER on GPUs. 2. Explicit Solvent Particle Mesh Ewald. J. Chem. Theory Comput. 2013; 9:3878-3888. [PubMed: 26592383]

45. Sugita Y, Okamoto Y. Replica-Exchange Molecular Dynamics Method for Protein Folding. Chem. Phys. Lett. 1999; 314:141-151.

46. Patriksson A, van der Spoel D. A Temperature Predictor for Parallel Tempering Simulations. Phys. Chem. Chem. Phys. 2008; 10:2073-2077. [PubMed: 18688361]

47. Lange OF, Grubmüller H. Full Correlation Analysis of Conformational Protein Dynamics. Proteins: Struct. Funct. Bioinforma. 2008; 70:1294-1312.

48. Grossfield, A. Version 2.0.9. Rochester, NY: University of Rochester; 2013. "WHAM: The Weighted Histogram Analysis Method.

49. Fiorin G, Klein ML, Hénin J. Using Collective Variables to Drive Molecular Dynamics Simulations. Mol. Phys. 2013; 111:3345-3362.

50. Phillips JC, Braun R, Wang W, Gumbart J, Tajkhorshid E, Villa E, Chipot C, Skeel RD, Kalé L, Schulten K. Scalable Molecular Dynamics with NAMD. J. Comput. Chem. 2005; 26:1781-1802. [PubMed: 16222654]

51. Mittermaier A, Kay LE. New Tools Provide New Insights in NMR Studies of Protein Dynamics. Science. 2006; 312:224-228. [PubMed: 16614210]

52. Vallurupalli P, Bouvignies G, Kay LE. Studying “Invisible” Excited Protein States in Slow Exchange with a Major State Conformation. J. Am. Chem. Soc. 2012; 134:8148-8161. [PubMed: 22554188]

53. Cozzi R, Malito E, Nuccitelli A, D’Onofrio M, Martinelli M, Ferlenghi I, Grandi G, Telford JL, Maione D, Rinaudo CD. Structure Analysis and Site-Directed Mutagenesis of Defined Key Residues and Motives for Pilus-Related Sortase C1 in Group B Streptococcus. FASEB J. 2011; 25:1874-1886. [PubMed: 21357525]

54. Cozzi R, Zerbini F, Assfalg M, D’Onofrio M, Biagini M, Martinelli M, Nuccitelli A, Norais N, Telford JL, Maione D, et al. Group B Streptococcus Pilus Sortase Regulation: A Single Mutation in the Lid Region Induces Pilin Protein Polymerization in Vitro. FASEB J. 2013; 27:3144-3154. [PubMed: 23631841]

55. Mazmanian SK, Ton-That H, Su K, Schneewind O. An Iron-Regulated Sortase Anchors a Class of Surface Protein during Staphylococcus Aureus Pathogenesis. Proc. Natl. Acad. Sci. U.S.A. 2002; 99:2293-2298. [PubMed: 11830639]

56. Race PR, Bentley ML, Melvin JA, Crow A, Hughes RK, Smith WD, Sessions RB, Kehoe MA, McCafferty DG, Banfield MJ. Crystal Structure of Streptococcus Pyogenes Sortase A. J. Biol. Chem. 2009; 284:6924-6933. [PubMed: 19129180]

57. Melvin JA, Murphy CF, Dubois LG, Thompson JW, Moseley MA, McCafferty DG. Staphylococcus Aureus Sortase A Contributes to the Trojan Horse Mechanism of Immune Defense 
Evasion with Its Intrinsic Resistance to Cys184 Oxidation. Biochemistry. 2011; 50:7591-7599. [PubMed: 21812416]

58. Wu C, Mishra A, Reardon ME, Huang I-H, Counts SC, Das A, Ton-That H. Structural Determinants of Actinomyces Sortase SrtC2 Required for Membrane Localization and Assembly of Type 2 Fimbriae for Interbacterial Coaggregation and Oral Biofilm Formation. J. Bacteriol. 2012; 194:2531-2539. [PubMed: 22447896] 
A

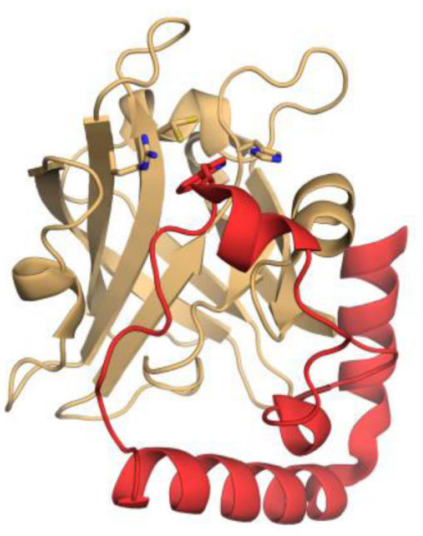

B

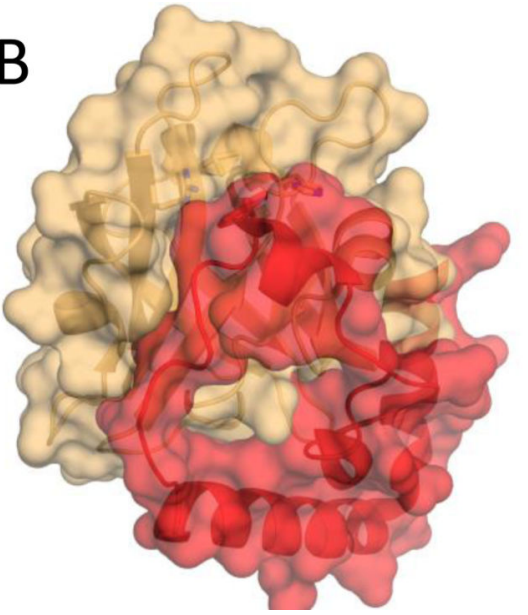

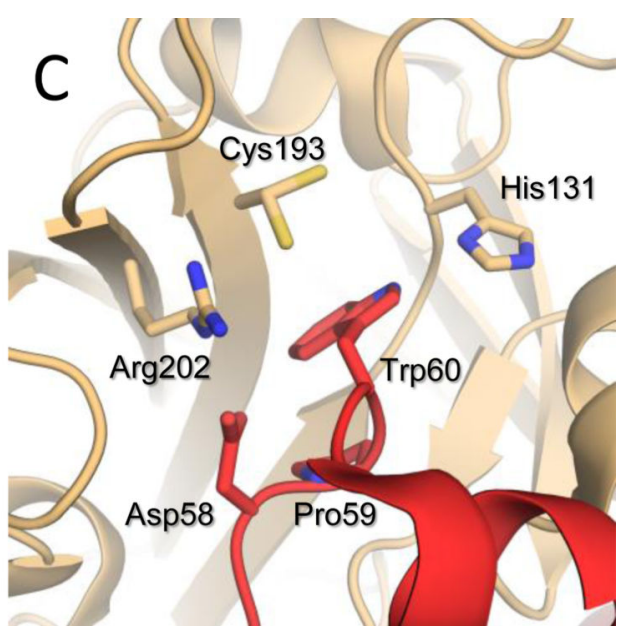

Figure 1. S. pneumoniae SrtC1 structure and lid motif (A) Cartoon representation of SrtC1 from PDB $2 \mathrm{~W} 1 \mathrm{~J}^{18}$. Active site His, Cys, Arg, and lid Trp residue shown as sticks. N-terminal extension preceding the sortase $\beta$-barrel core is colored red. (B) Transparent surface representation of $\mathrm{SrtC}$, showing the lid occluding the active site. (C) Zoom in of SrtC1 active site, showing conserved DP(F/W/Y) lid motif in active site as sticks. 

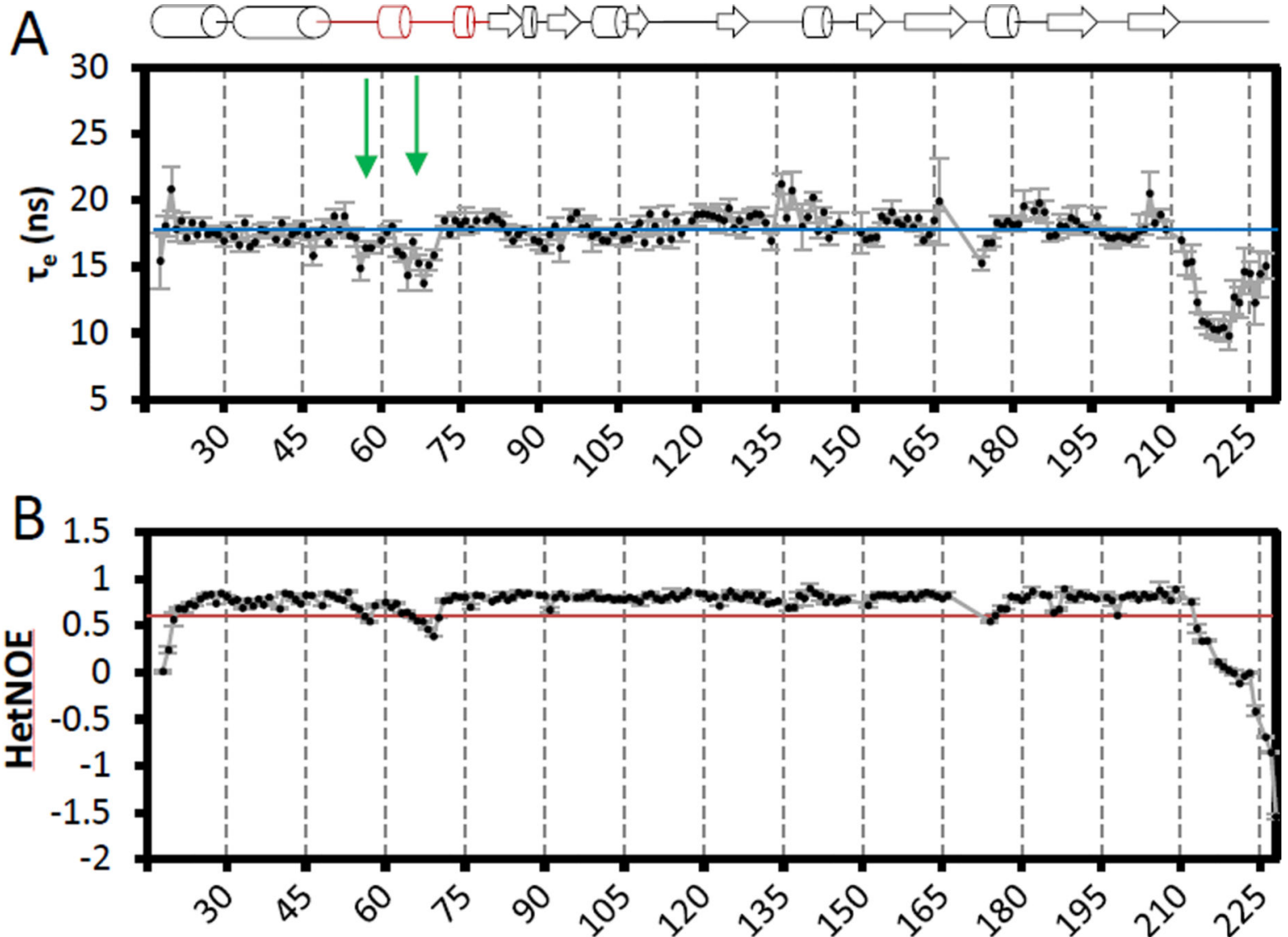

C

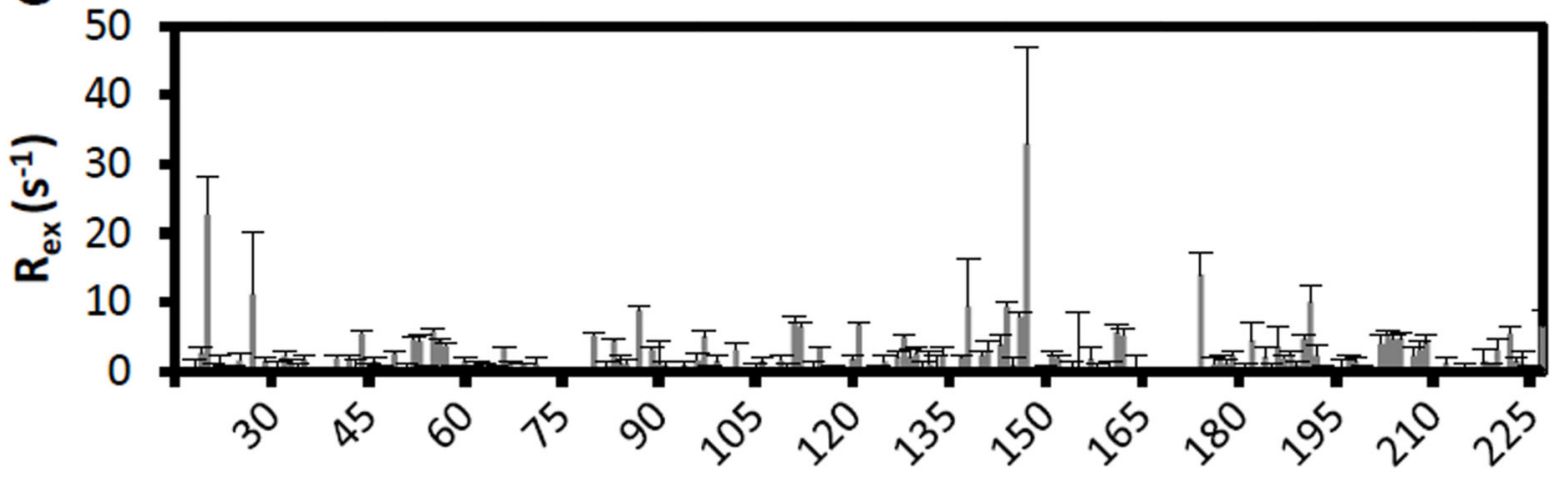

\section{Residue}

Figure 2.

NMR dynamics of SrtC1. A diagram of secondary structure elements is shown at the top of the figure where helices are represented as cylinders and $\beta$-strands as arrows, with the lid region colored red. (A) Residue specific tumbling time $\left(\boldsymbol{\tau}_{\mathrm{e}}\right)$ for each assigned residue in $\mathrm{SrtC1}$, with dynamic regions of the lid indicated by green arrows. Average for the structured region of the protein plotted as a blue line. (B) Heteronuclear NOE for each residue in SrtC1. A red line is shown at a HetNOE value of 0.6. A HetNOE value below this line 
indicates that the residue can be considered dynamic. (C) $\mathrm{R}_{\mathrm{ex}}$ component of relaxation as determined by direct CPMG detection, plotted for each residue. 

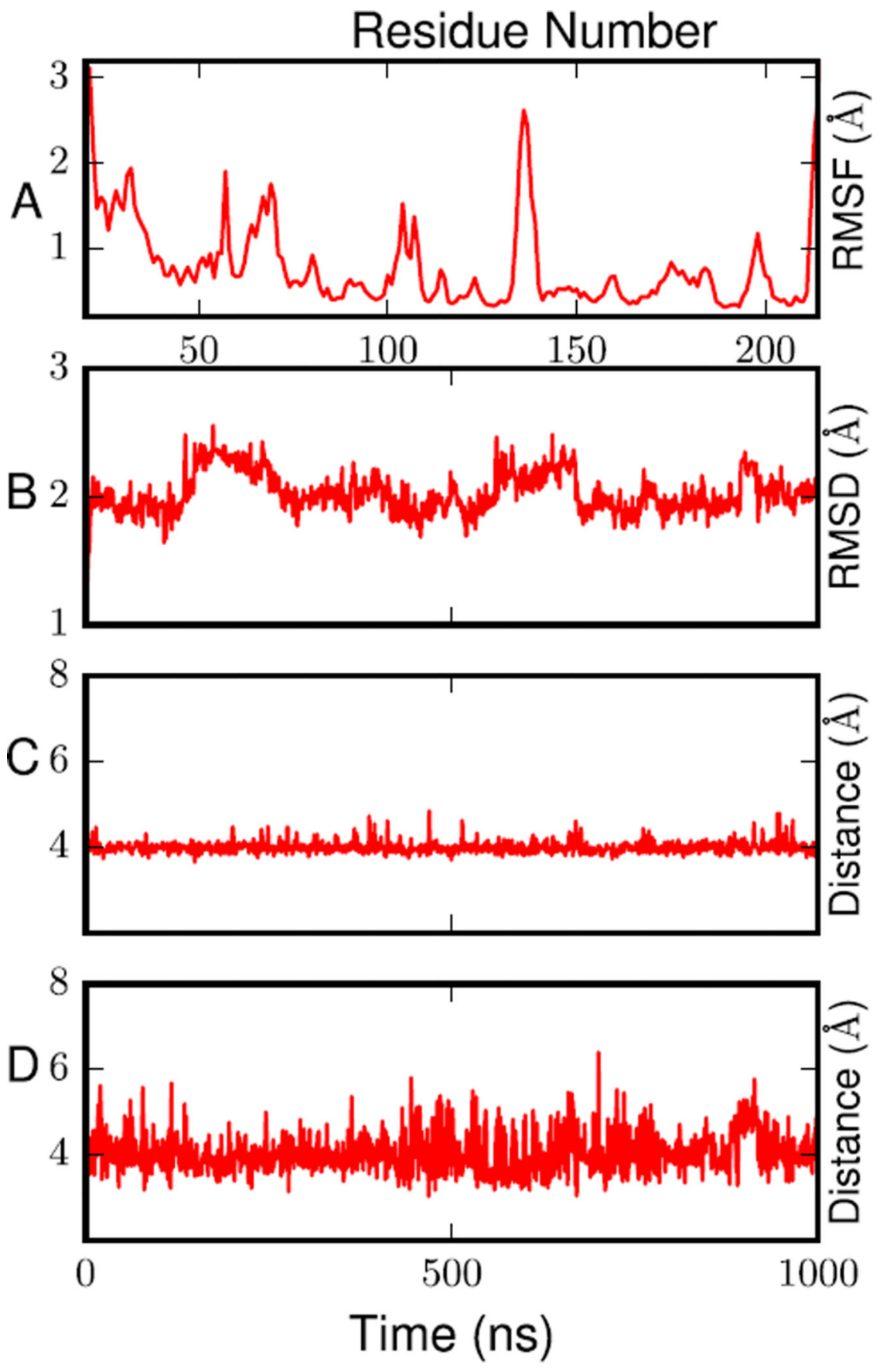

Figure 3.

Dynamical characteristics of wild-type $\mathrm{SrtC} 1$ obtained from $1000 \mathrm{~ns}$ cMD simulations including (A) root mean squared fluctuations (RMSF) of the $\mathrm{C}_{a}$ atoms, (B) root mean squared deviations of the $\mathrm{C}_{\alpha}$ atoms from their crystal structure positions, (C) distance between the $\mathrm{CZ}$ atom of R202 and the CG atom of residue 58 (salt bridge distance) and (D) The distance between the SG atom of residue $\mathrm{C} 193$ and the $\mathrm{CD} 2$ atom of residue W60 (Sulphur-aromatic interaction). 

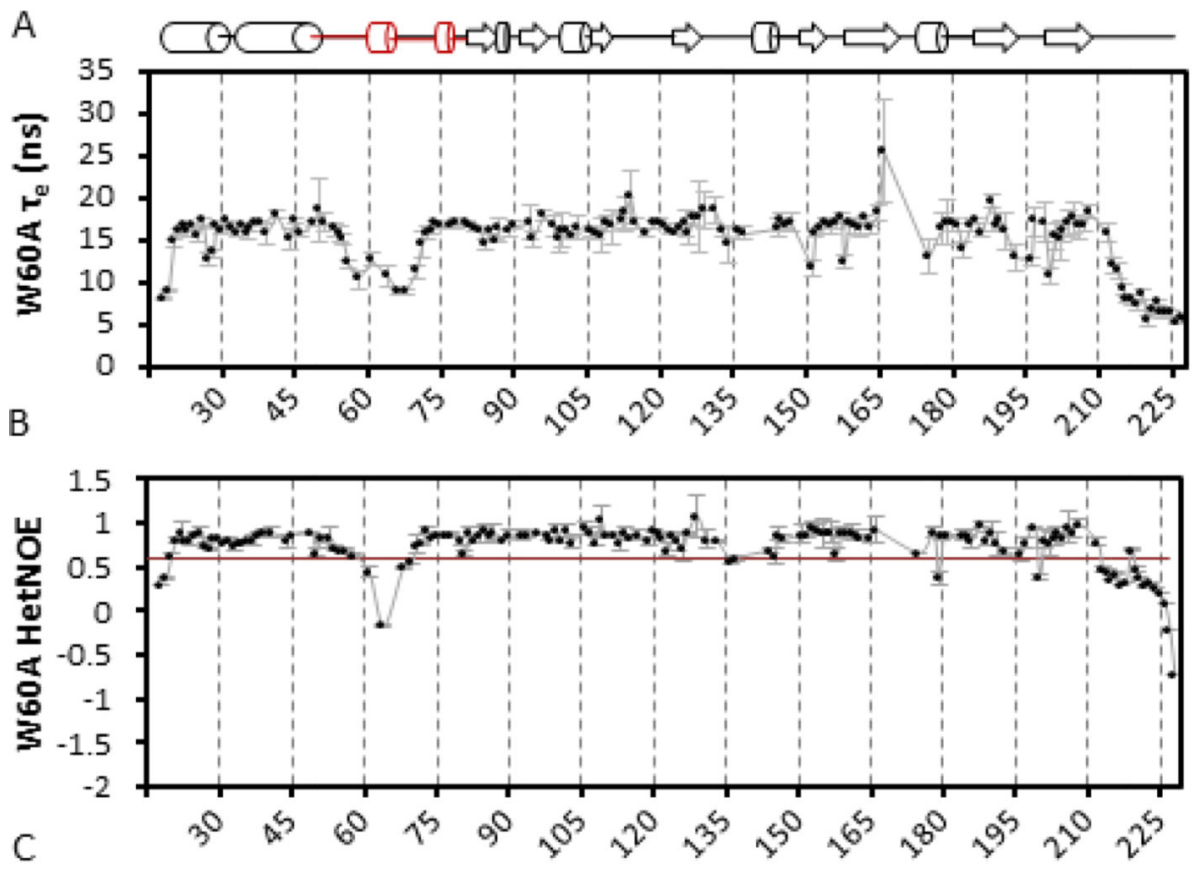

E
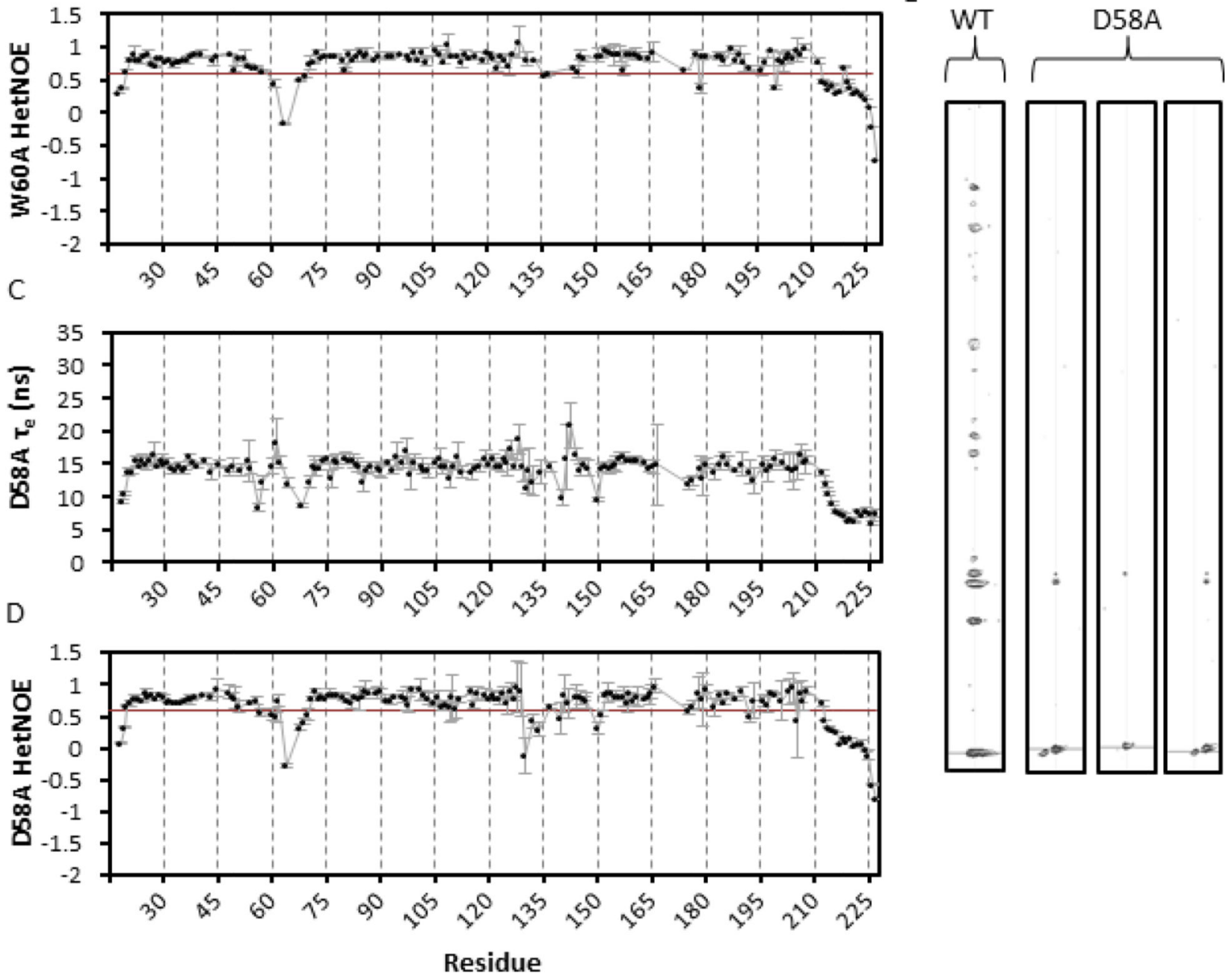

Figure 4.

NMR shows SrtC1 mutants have more dynamic lids. A diagram of secondary structure elements is shown at the top where helices are represented as cylinders and $\beta$-strands as arrows, with the lid region highlighted in red. (A) Residue specific tumbling time $\left(\tau_{\mathrm{e}}\right)$ for each assigned residue in SrtC1-W60A. (B) Heteronuclear NOE for each residue in SrtC1W60A. Red line at 0.6 indicates the cutoff below which residues are considered disordered (C) Residue specific tumbling time $\left(\boldsymbol{\tau}_{\mathrm{e}}\right)$ for each assigned residue in SrtC1-D58A. (D) Heteronuclear NOE for each residue in SrtC1-D58A. Red line at 0.6 indicates the cutoff 
below which residues are considered disordered. (E) Comparison of NOESY spectra for the W60 indole proton for the WT SrtC1 protein, left, or three possible indole configurations seen in the D58A mutant, represented by the right 3 strips. 

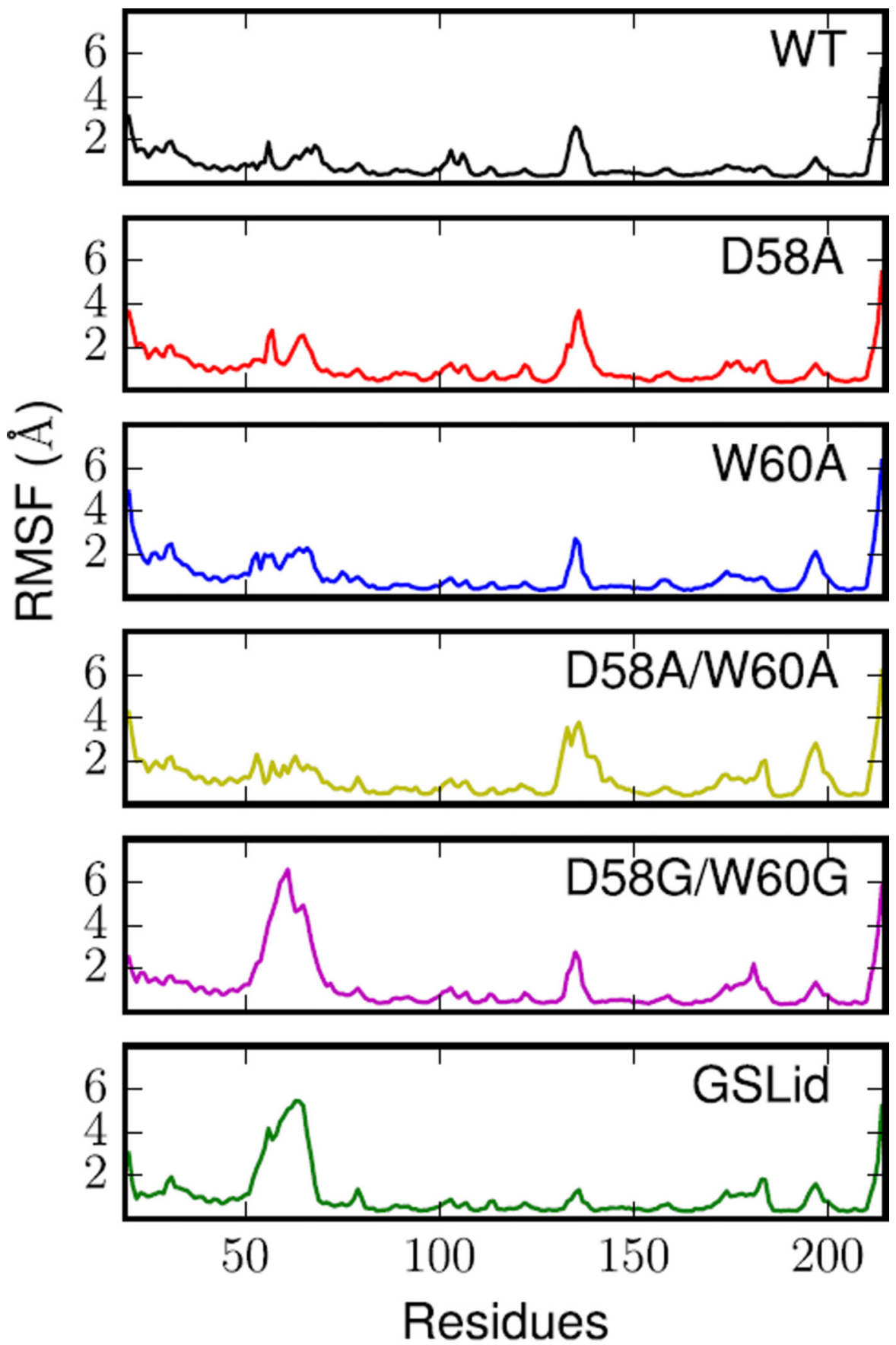

Figure 5.

Root mean squared fluctuations (RMSF) of the $\mathrm{C}_{\mathrm{a}}$ atoms of SrtC1 shows that the lid region of the wild-type protein is more rigid in comparison with the mutants discussed in this study. The lid region of the D58G/W60G and GSlid mutants adopt particularly open conformations with RMSF values around $6 \AA$. 


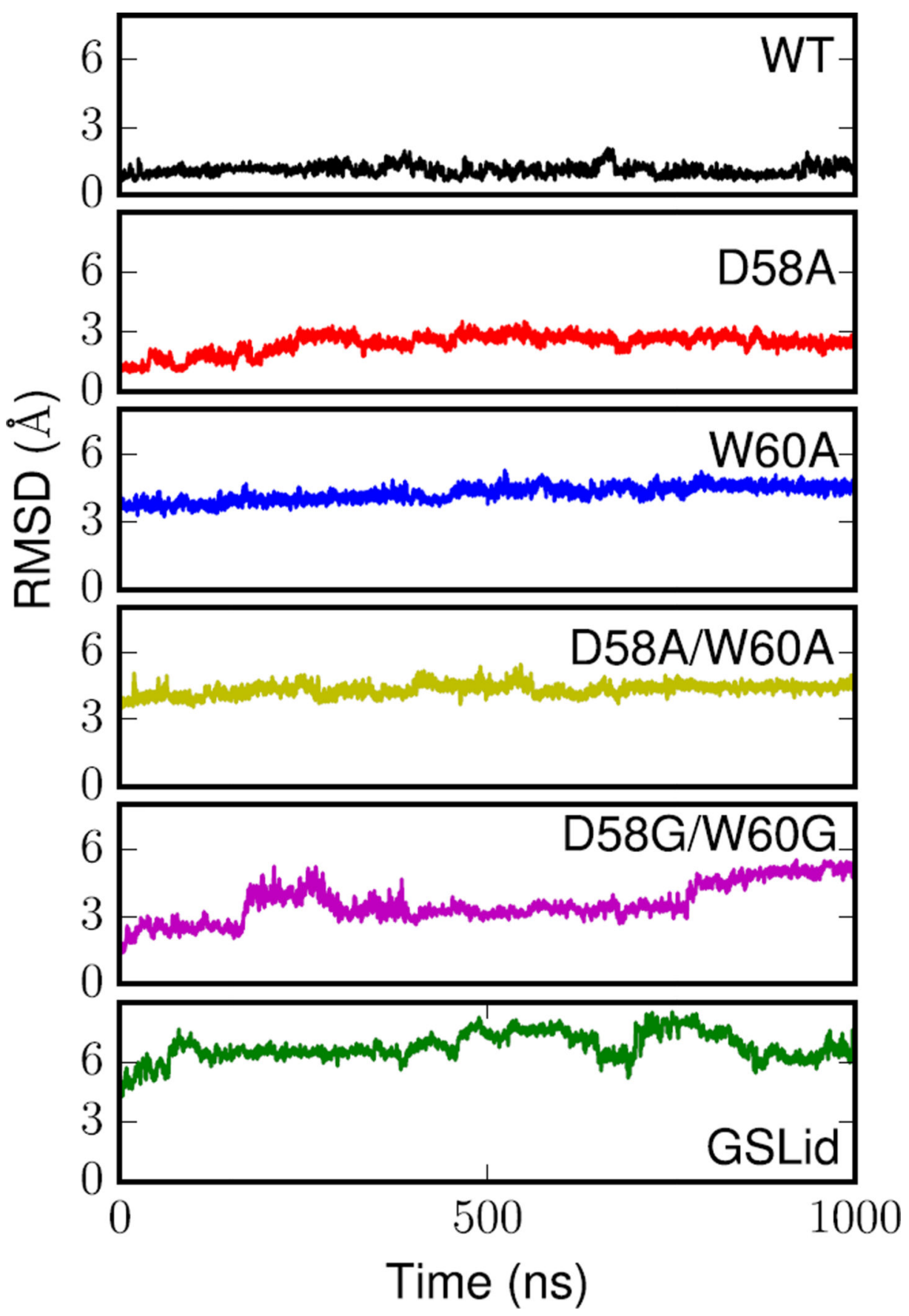

Figure 6.

Root mean squared deviations (RMSD) of the $\mathrm{C}_{a}$ atoms of the lid region (residues 50 to 70) for all proteins considered in this study. All mutants show elevated values compared to the wild-type with more pronounced deviations for D58G/W60G and GSlid as seen in the RMSF plots. 


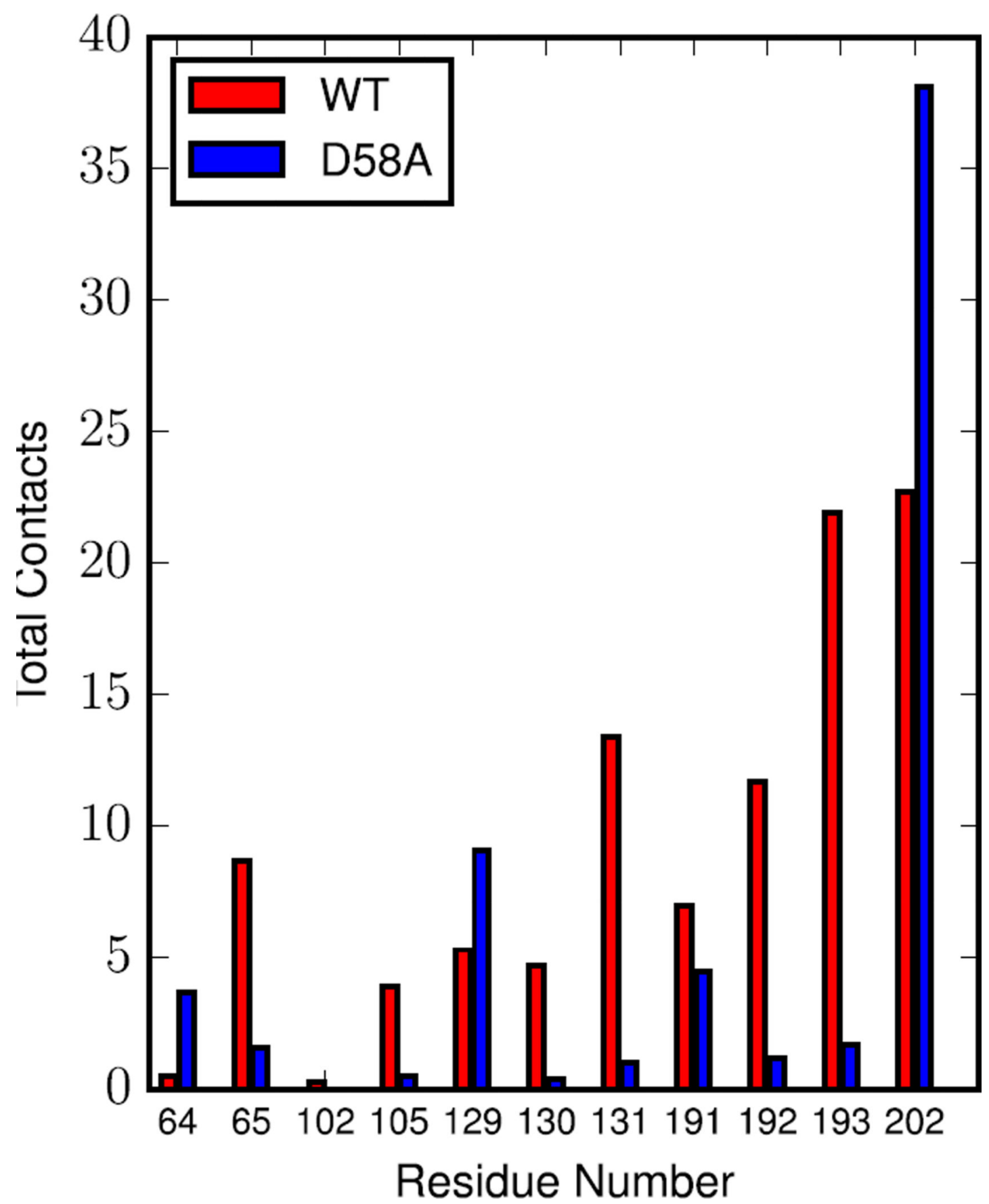

Figure 7.

Total of all contact fractions between atoms in the side chain of residue W60 of D58A compared to $\mathrm{SrtC}$. The side chain is docked into a hydrophobic grove formed by residues upstream from the active site leading to a different molecular environment compared to its conformation in the wild type. 

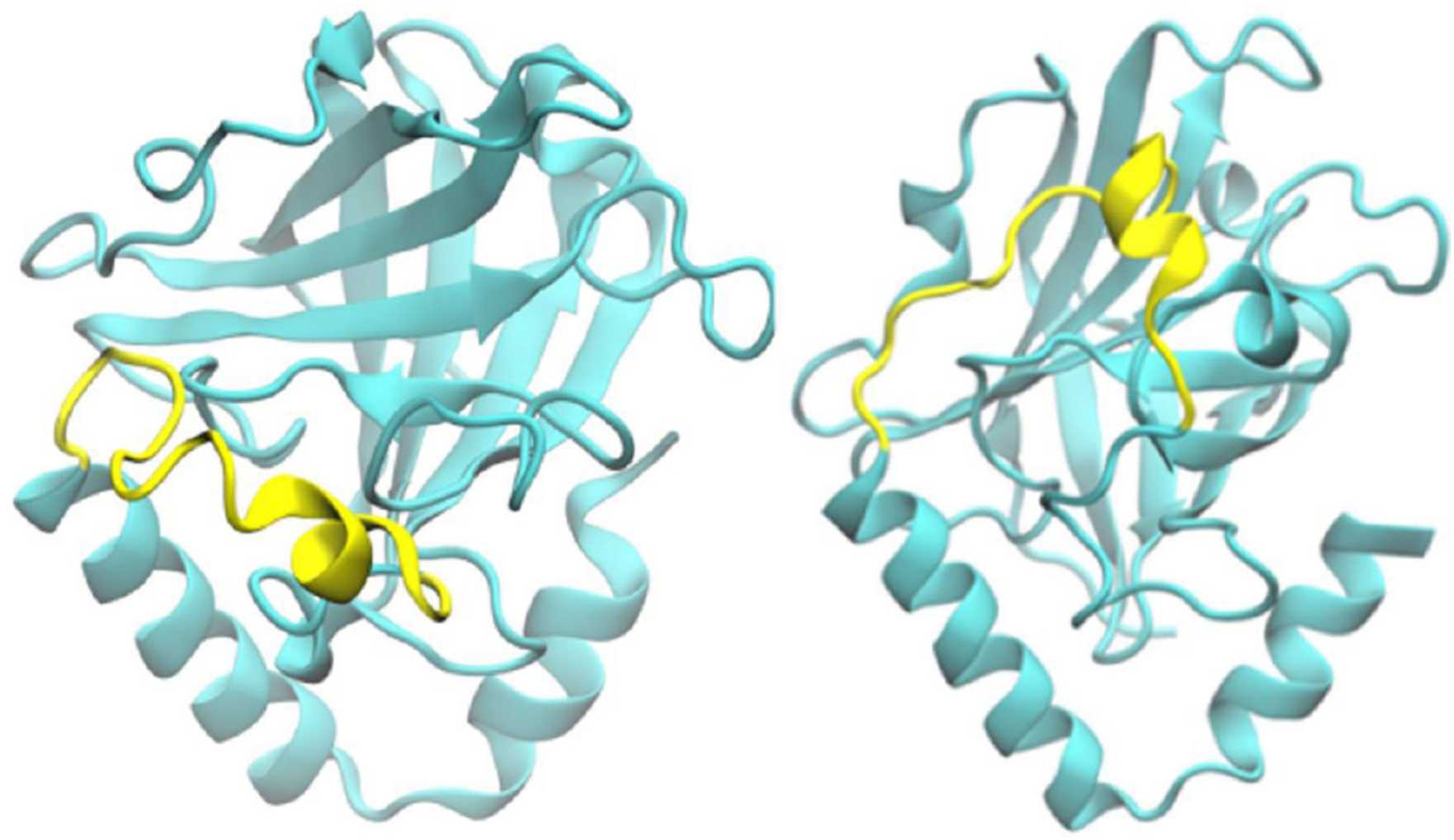

Figure 8.

Open (left) and closed (right) conformations of the lid observed in cMD calculations of the GS-linker mutant of SrtC1. 


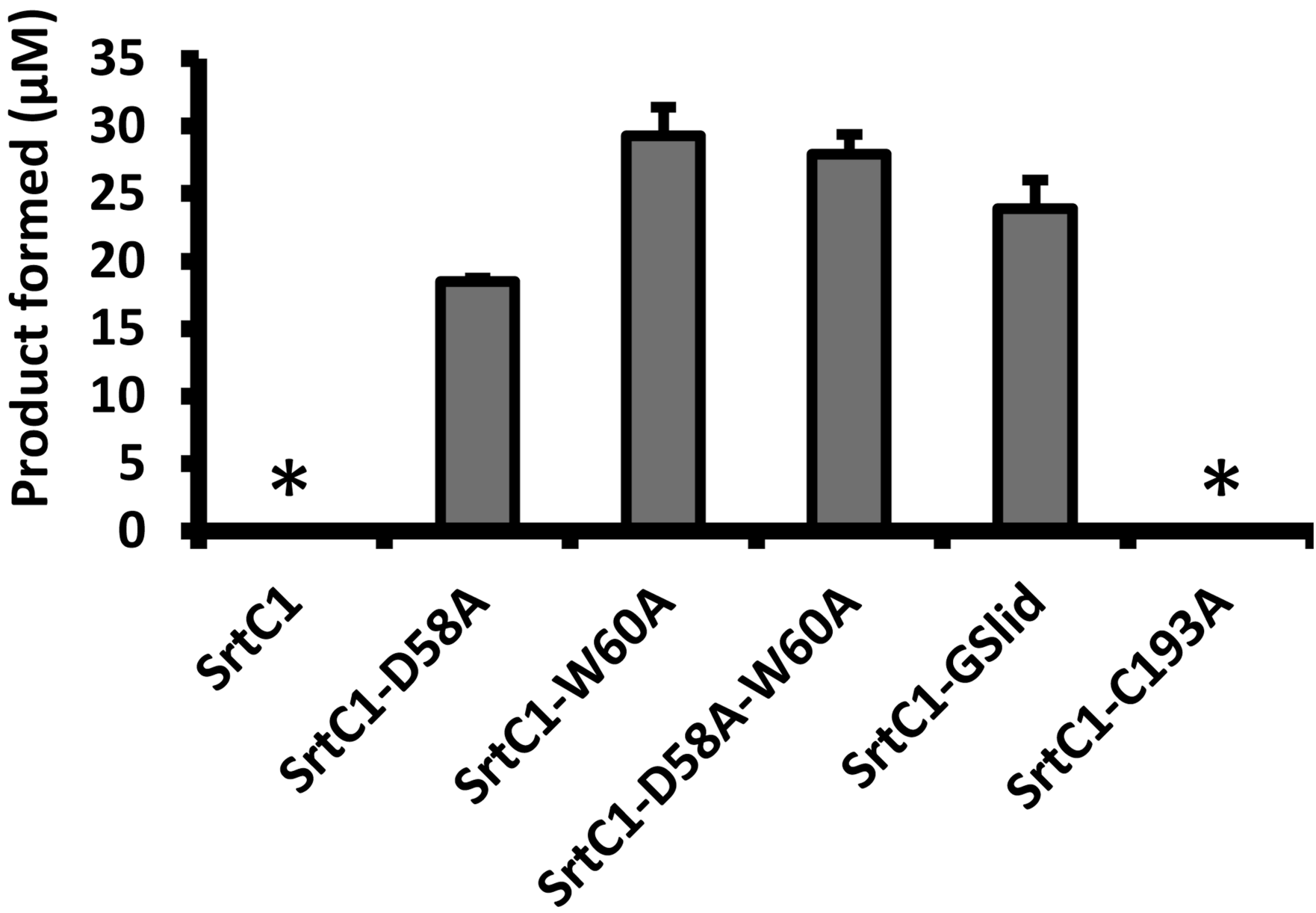

Figure 9.

$\mathrm{SrtC} 1$ lid mutants are more active in vitro. Product formed after $24 \mathrm{hr}$ reactions with IPQTG containing peptide is shown for WT, and various SrtC1 mutant constructs. * indicates no product could be detected by HPLC or MALDI. $p<0.01$ for each mutant compared to WT, except C193A. 


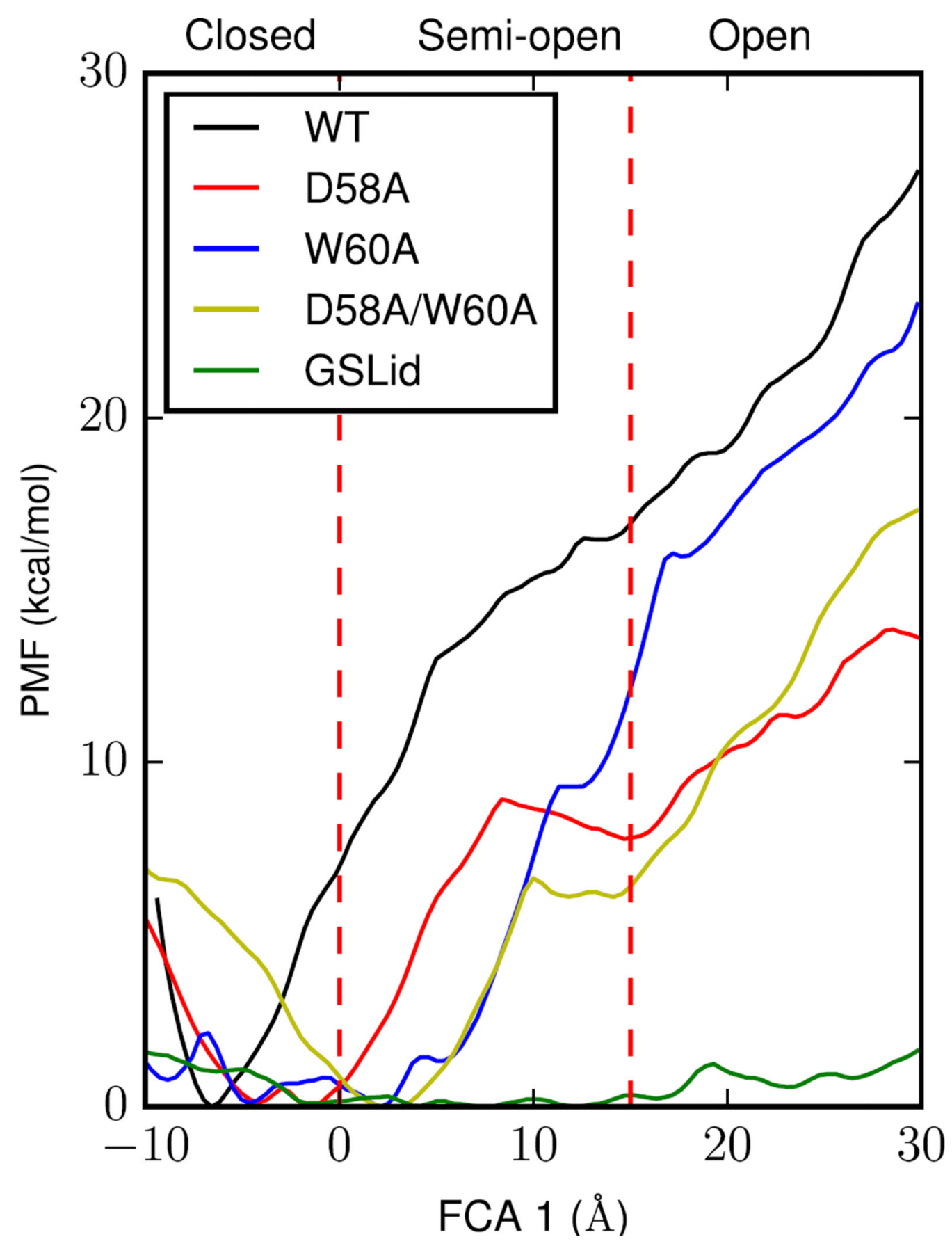

Figure 10.

Potential of mean force (PMF) for lid opening for SrtC1 and its mutants. Regions corresponding to open, semi-open and closed states are indicated with dashed lines. It can be seen that the WT SrtC11 requires the highest free energy to access semi-open and open states. 